Nonlinear Identification of a Physically Parameterized Robot Model

\author{
Erik Wernholt, Svante Gunnarsson
}

Division of Automatic Control

Department of Electrical Engineering

Linköpings universitet, SE-581 83 Linköping, Sweden

WWW: http://www.control.isy.liu.se

E-mail: erikw@isy.liu.se, svante@isy.liu.se

29th August 2006

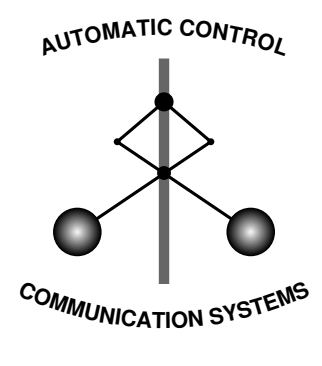

LINKÖPING

Report no.: LiTH-ISY-R-2739

Accepted for publication in SYSID 2006, Newcastle, Australia

Technical reports from the Control \& Communication group in Linköping are available at http://www.control.isy.liu.se/publications. 


\begin{abstract}
In the work presented here, a three-step identification procedure for rigid body dynamics, friction, and flexibilities, introduced in (Wernholt and Gunnarsson, 2005), will be utilized and extended. Using the procedure, the parameters can be identified only using motor measurements. In the first step, rigid body dynamics and friction will be identified using a separable least squares method, where a friction model describing the Striebeck effect is used. In the second step, initial values for flexibilities are obtained using inverse eigenvalue theory. Finally, in the last step, the remaining parameters of a nonlinear physically parameterized model are identified directly in the time domain. The procedure is exemplified using real data from an experimental industrial robot.
\end{abstract}

Keywords: Identification, Robotics, Flexible arms, Friction, Manipulators 


\title{
NONLINEAR IDENTIFICATION OF A PHYSICALLY PARAMETERIZED ROBOT MODEL ${ }^{1}$
}

\author{
Erik Wernholt and Svante Gunnarsson
}

\author{
Dept. of Electrical Engineering, Linköping University, \\ SE-58183 Linköping, Sweden
}

\begin{abstract}
In the work presented here, a three-step identification procedure for rigid body dynamics, friction, and flexibilities, introduced in (Wernholt and Gunnarsson, 2005), will be utilized and extended. Using the procedure, the parameters can be identified only using motor measurements. In the first step, rigid body dynamics and friction will be identified using a separable least squares method, where a friction model describing the Striebeck effect is used. In the second step, initial values for flexibilities are obtained using inverse eigenvalue theory. Finally, in the last step, the remaining parameters of a nonlinear physically parameterized model are identified directly in the time domain. The procedure is exemplified using real data from an experimental industrial robot.
\end{abstract}

Keywords: Identification, Robotics, Flexible arms, Friction, Manipulators

\section{INTRODUCTION}

System identification in robotics is a vast research area and can be divided into, at least, three different levels or application areas. These levels involve the estimation of the kinematic description, the dynamic model (often divided into rigid body and flexible body dynamics), and the joint model (e.g., motor inertia, gearbox elasticity and backlash, motor characteristics, and friction parameters). Some results on the latter two areas are mentioned in Section 4. An overview of identification in robotics can also be found in (Kozlowski, 1998).

The work reported here consider nonlinear identification of rigid body dynamics, friction, and flexibilities using a three-step procedure first introduced in (Wernholt and Gunnarsson, 2005). The procedure is extended both regarding the friction model, here leading to a pseudo-linear regression, and the example now have a nonlinear three-mass model. The work is also closely related to the problems considered in, for

\footnotetext{
1 Supported by VINNOVA's Center of Excellence ISIS at Linköping University.
}

example, (Östring et al., 2003; Isaksson et al., 2003). In (Östring et al., 2003), a method is applied where inertial parameters as well as parameters describing the flexibility of a three-mass model can be identified directly in the time domain. However, only linear models were considered in their work. (Isaksson et al., 2003) consider grey-box identification of a twomass model with backlash, where black-box modeling is used to find initial parameter values.

\section{NONLINEAR GREY-BOX IDENTIFICATION}

The starting point for the nonlinear grey-box identification is the continuous-time state space model structure

$$
\begin{aligned}
& \dot{x}(t)=f(t, x(t), \theta, u(t)) \\
& y(t)=h(t, x(t), \theta, u(t))+e(t)
\end{aligned}
$$

where $f$ and $h$ are nonlinear functions. $x(t)$ is the state vector, $u(t)$ and $y(t)$ are input and output signals, $e(t)$ a white measurement disturbance signal, and $t$ denotes time. Finally $\theta$ is the vector of unknown parameters. Given a set of input/output-data the aim 
is to determine the parameter vector that minimizes a criterion like

$$
V_{N}(\theta)=\frac{1}{N} \sum_{t=1}^{N} \varepsilon^{2}(t, \theta)
$$

where $\varepsilon(t)$ denotes the prediction error

$$
\varepsilon(t, \theta)=y(t)-\hat{y}(t, \theta)
$$

The experiments presented in this paper will utilize the nonlinear grey-box model structure NLGREY, available in a beta version of a nonlinear extension to the System Identification Toolbox (SITB), (Ljung, 2003). The model structure NLGREY is similar to the IDGREY model structure in SITB. The model can be either a discrete-time or continuous-time state space model, and it is defined in a Matlab m-file/mex-file. In the current version of the software, only OE-models can be used, i.e. only additive white noise, $e(t)$, on the output. The prediction $\hat{y}(t \mid \theta)$ then becomes the simulated output of the model (1) with the input $u(t)$, without $e(t)$, for the current parameter vector $\theta$. The criterion (2) is minimized by an iterative numerical search algorithm, which involves simulation of the system for different values of $\theta$. The user specifies an initial parameter vector and it is also possible to fix some components in $\theta$. To speed up the numerical optimization, the simulation model is implemented in a mex-file (C-code).

\section{ROBOT MODEL}

The industrial robot that will be studied in this paper is, for movements around an axis not affected by gravity, modeled by a nonlinear three-mass flexible model which is illustrated in Figure 1. Gear ratio $r=1$ is used in the model, which gives better numerical properties and easier notation. The true physical parameters can later be obtained by a simple scaling with the true gear ratio.

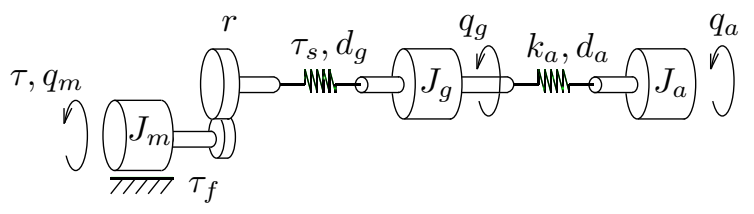

Fig. 1. The three-mass flexible model of the robot arm.

The input is the torque $\tau$ generated by the electrical motor, while the output is the motor velocity $\dot{q}_{m}$. The velocities of the other masses, $\dot{q}_{g}$ and $\dot{q}_{a}$, are not measurable. Flexibility in the gearbox is modeled by a nonlinear spring, $\tau_{s}(\cdot)$, between the motor and the second mass. The spring between the second and third mass represents flexibilities in the arm structure. Finally, friction in the system is modeled by nonlinear friction, $\tau_{f}(\cdot)$, acting on the first mass.
Applying torque balances for the three masses and introducing the states

$$
x(t)=\left(\begin{array}{c}
q_{m}(t)-q_{g}(t) \\
q_{g}(t)-q_{a}(t) \\
\dot{q}_{m}(t) \\
\dot{q}_{g}(t) \\
\dot{q}_{a}(t)
\end{array}\right)
$$

gives the nonlinear state-space model

$$
\dot{x}=\left(\begin{array}{c}
x_{3}-x_{4} \\
x_{4}-x_{5} \\
\frac{1}{J_{m}}\left(-\tau_{s}\left(x_{1}\right)-d_{g}\left(x_{3}-x_{4}\right)-\tau_{f}\left(x_{3}\right)+u\right) \\
\frac{1}{J_{g}}\left(\tau_{s}\left(x_{1}\right)+d_{g}\left(x_{3}-x_{4}\right)-k_{a} x_{2}-d_{a}\left(x_{4}-x_{5}\right)\right) \\
\frac{1}{J_{a}}\left(k_{a} x_{2}+d_{a}\left(x_{4}-x_{5}\right)\right)
\end{array}\right)
$$

$y=x_{3}$

where $J_{m}, J_{g}$, and $J_{a}$ are the moments of inertia of the motor, gear, and arm respectively, $u=\tau$ is the motor torque, $d_{g}$ and $d_{a}$ are damping parameters, and $k_{a}$ is the stiffness of the second spring. The spring and gear friction torques, $\tau_{s}$ and $\tau_{f}$ respectively, are often approximately modeled by linear models (see, for example, (Östring et al., 2003)). In this work, a nonlinear friction model will be used to capture Coulomb friction and the Striebeck effect as

$$
\tau_{f}\left(x_{3}\right)=F_{v} x_{3}+\left(F_{c}+F_{c s} \operatorname{sech}\left(\alpha x_{3}\right)\right) \tanh \left(\beta x_{3}\right)
$$

where $F_{v}$ and $F_{c}$ are the viscous and Coulomb friction coefficients, $F_{c s}$ and $\alpha$ are used to model the Striebeck effect, and $\beta$ is used to get a smooth model without discontinuity at zero velocity, which is more suitable for simulation. As a comparison, the simpler friction model

$$
\tau_{f}\left(x_{3}\right)=F_{v} \dot{x}_{3}+F_{c} \operatorname{sgn}\left(x_{3}\right)
$$

will be used as well. For details on friction modeling, see (Armstrong-Hélouvry et al., 1994). The torque of the spring is modeled as

$$
\tau_{s}\left(x_{1}\right)=k_{g 1} x_{1}+k_{g 3} x_{1}^{3}
$$

where $k_{g 1}$ and $k_{g 3}$ are the parameters of the spring.

\section{IDENTIFICATION PROCEDURE}

The aim is to identify all parameters in the robot model, described in Section 3, using experimental data and the nonlinear grey-box identification procedure described in Section 2. An inherent problem of iterative search routines is that only convergence to a local minimum can be guaranteed. In order to converge to the global minimum, a good initial parameter estimate is important. Therefore a three-step identification procedure is proposed. The first step consider identification of rigid body dynamics and friction. In the second step, initial values for flexibilities are obtained. Finally, in the third step the nonlinear grey-box identification procedure is applied. 


\subsection{Step 1: Rigid body dynamics and friction}

There exists a vast amount of literature on the identification of rigid body dynamics, see, for example, (Grotjahn et al., 2001; Gautier and Poignet, 2001; Kozlowski, 1998; Swevers et al., 1997). The standard procedure includes a dynamic model

$$
H_{r b}(q, \dot{q}, \ddot{q}) \theta_{r b}=\tau
$$

which is linear in the rigid body parameters $\theta_{r b}$. Each link gives ten physical parameters. This representation is redundant, but there are methods to find a minimal dimensional parameter vector $\theta_{b}$, called base parameters, that characterize the dynamic model like

$$
H_{b}(q, \dot{q}, \ddot{q}) \theta_{b}=\tau
$$

The base parameters are nonlinear functions of the physical parameters like

$$
\theta_{b}=\phi\left(\theta_{r b}\right)
$$

In the presence of friction, $\tau$ in (11) should be replaced by $\tau-\tau_{f}(\dot{q})$. Usually the friction model (8) is used, which gives two additional parameters per link to estimate, but still a linear regression. This model is not sufficient to correctly describe dynamic friction, see (Armstrong-Hélouvry et al., 1994), but compensates the major frictional effects on the identification of rigid body dynamics when using a high amplitude excitation. The robot is moved along some (optimized) trajectory and applied torque and joint movements are recorded. The parameters are then estimated using linear regression.

Here, the more advanced friction model (7) will be used in order to more accurately model the low velocity behavior. For the robot model in Section 3, the rigid body dynamics and friction is

$$
\left(J_{m}+J_{g}+J_{a}\right) \ddot{q}_{m}+\tau_{f}\left(\dot{q}_{m}\right)=\tau
$$

which can be written as a pseudo-linear regression

$$
\hat{\tau}(t \mid \rho, \eta)=\rho^{T} \varphi(t, \eta)
$$

when

$$
\begin{aligned}
\varphi(t, \eta) & =\left(\begin{array}{c}
\ddot{q}_{m} \\
\dot{q}_{m} \\
\tanh \left(\beta \dot{q}_{m}\right) \\
\operatorname{sech}\left(\alpha \dot{q}_{m}\right) \tanh \left(\beta \dot{q}_{m}\right),
\end{array}\right) \\
\rho & =\left(\begin{array}{lllll}
J & F_{v} & F_{c} & F_{c s}
\end{array}\right)^{T}, \quad \eta=(\alpha \beta)^{T} .
\end{aligned}
$$

Here, $J$ is the only base parameter and (12) simplifies to $J=J_{m}+J_{g}+J_{a}$.

Minimizing an identification criterion like

$$
V_{N}(\rho, \eta)=\sum_{t=1}^{N}\left|\tau(t)-\rho^{T} \varphi(t, \eta)\right|^{2}=|\boldsymbol{\tau}-\Phi(\eta) \rho|^{2}
$$

then is a separable least squares problem since the least squares part $\rho$ can be separated out using

$$
\hat{\rho}=\left[\Phi^{T}(\eta) \Phi(\eta)\right]^{-1} \Phi^{T}(\eta) \tau
$$

and the problem is reduced to finding the optimal $\eta$. See (Golub and Pereyra, 1973) for a thorough treatment of this approach.

The result from the first step are estimates of the base parameters $\hat{\theta}_{b}$ and the friction parameters $\hat{\theta}_{f r}$.

\subsection{Step 2: Initial values for flexibilities}

The major flexibility in an industrial robot is normally located at the joint level, due to the transmission. A two-mass model (or coupled two-mass models for multivariable cases) is then sufficient to describe the dynamics. Weaker (more compliant) robot structures will in addition introduce significant flexibilities in the links and their connections. Therefore higher order models are sometimes needed in order to get a sufficient description of the system. Identification of flexibilities is more involved than the identification of rigid body dynamics. The main reason is that now typically only a subset of the state variables are measured and one can therefore not use linear regression. Many different methods are described in the literature to handle this problem, see for example (Behi and Tesar, 1991; Johansson et al., 2000; Albu-Schäffer and Hirzinger, 2001). They differ in, for example, assumed model structure, required measurement signals, and complexity of the identification method.

Here, a method described in (Berglund and Hovland, 2000) will be used for the identification of masses, springs and dampers, only using applied torque and joint movements. The identification is based on an estimated Frequency Response Function (FRF) together with the solution of an inverse eigenvalue problem. Consider an Nth order spring-mass system, which can be modeled as

$$
M \ddot{q}+K q=\tau
$$

where $M$ is a diagonal inertia matrix, $q=\left(q_{1} \ldots q_{N}\right)^{T}$ is a vector of the position of the masses, and $K$ is a tridiagonal matrix built up from the $N-1$ spring constants. The eigenvalues of the system are given by

$$
B u=\lambda u
$$

where $B=L^{-1} K L^{-T}, u=L^{T} q$, and $L=M^{1 / 2}$. Using the FRF gives information about the resonance frequencies and anti-resonance frequencies. The first eigenvector $u_{1}$ of $B$ can be derived from these frequencies, assuming that the damping is neglectable. The $B$ matrix can then be computed recursively by the Lanczos algorithm using $u_{1}$ and the squared resonance frequencies. Finally, the elements of $M$ and $K$ can be reconstructed by using their special structure. A damping matrix can be estimated afterwards by minimizing the distance between the FRF and the model at the resonance and anti-resonance frequencies, using some optimization routine. For details about the method, see (Berglund and Hovland, 2000) and the references therein. 
The result from the second step gives initial estimates of (some) rigid body parameters, $\hat{\theta}_{r b}$, and parameters describing the flexibilities, $\theta_{f l}$, typically springs and dampers. As an optional step, one could of course refine all these estimated parameters by curve fitting between the FRF and the model as well.

\subsection{Step 3: Nonlinear grey-box identification}

Combining the estimates from step 1 and 2 gives an initial parameter estimate, and the nonlinear greybox identification method described in Section 2 can now be applied. To reduce the complexity, parameters estimated with high accuracy in the previous steps can be fixed in this step, leading to a lower dimensional iterative search. Keeping $\hat{\theta}_{b}$ and $\hat{\theta}_{f r}$ from step 1 fixed will result in a modified criterion

$$
\begin{aligned}
\min _{\theta} V_{N}(\theta) & =\frac{1}{N} \sum_{t=1}^{N} \varepsilon^{2}(t, \theta) \\
\text { subject to } \phi\left(\theta_{r b}\right)-\hat{\theta}_{b} & =0 \\
\theta_{f r}-\hat{\theta}_{f r} & =0
\end{aligned}
$$

where $\theta=\left(\begin{array}{lll}\theta_{r b}^{T} & \theta_{f r}^{T} & \theta_{f l}^{T}\end{array}\right)^{T}$.

\section{DATA COLLECTION}

The data used for identification are real data collected from an experimental robot. For this kind of application it is necessary to use feedback control while data are collected, both for safety reasons and in order to keep the robot around its operation point. An experimental control system is used, which makes it possible to use off-line computed reference signals for the joint controllers.

For the different steps in the identification procedure, different excitation signals are needed. In step 1, the rigid body dynamics and friction parameters should be excited without introducing any oscillations due to the flexibilities. Therefore a low frequency excitation is preferred. In step 2, on the other hand, the whole frequency band should be excited where notch and peak frequencies in the frequency response function are expected. Since FRF estimates are based on an assumption of linearity, the influence of nonlinear friction should reduced, so a broadband excitation with as few zero velocity crossings as possible is selected. Finally, for step 3 a data set (or a combination of data sets) is needed that excite all free parameters in the model. The following periodic excitation signals will be used as reference speed, $\dot{q}_{m}^{r e f}$, for the controller. They all have a period time of $10 \mathrm{~s}$ and one period the steady state response is collected, sampled at $2 \mathrm{kHz}$ ( $T=0.5 \mathrm{~ms})$.

Data set 1: Multisine signal (sum of sinusoids) with frequencies $0.1,0.3$, and $0.5 \mathrm{~Hz}$. Five different peak values have been applied: 1, 5, 20, 40, and $160 \mathrm{rad} / \mathrm{s}$.
Table 1. Estimated parameters from step 1, including one standard deviation.

\begin{tabular}{llll}
\hline$J$ & $0.0329 \pm 0.0027$ & $F_{c s}$ & $3.9863 \pm 4.5876$ \\
$F_{v}$ & $0.0099 \pm 0.0074$ & $\alpha$ & $3.2402 \pm 0.5469$ \\
$F_{c}$ & $0.7430 \pm 0.3774$ & $\beta$ & $0.7994 \pm 0.2685$ \\
\hline
\end{tabular}

Table 2. Loss function when evaluating different friction models on data set 1 with different amplitudes.

\begin{tabular}{lllll}
\hline Amplitudes: & 1 & 5 & 20 & 40 \\
\hline (7) & 0.0794 & 0.0458 & 0.0287 & 0.0439 \\
$(7), F_{c s}=0$ & 0.0897 & 0.0406 & 0.0292 & 0.0443 \\
$(8)$ & 0.1603 & 0.0475 & 0.0275 & 0.0449 \\
\hline
\end{tabular}

Data set 2: Multisine signal with a flat amplitude spectrum in the frequency interval $1-40 \mathrm{~Hz}$ with a peak value of $16 \mathrm{rad} / \mathrm{s}$. The multisine signal is superimposed on a filtered square wave with amplitude $20 \mathrm{rad} / \mathrm{s}$ and cut-off frequency $1 \mathrm{~Hz}$.

Data set 3: Similar to data set 2, but without the square wave.

For details on the selection of excitation signals, see, e.g. (Pintelon and Schoukens, 2001; Ljung, 1999).

\section{RESULTS}

The physical parameters in the robot model from Section 3 will here be identified by applying the proposed three-step identification procedure from Section 4, using the experimental data described in Section 5.

\subsection{Step 1}

Using data set 1 , with one period of data for each of the five amplitudes, together with the separable least squares method, as described in Section 4.1, gives parameter estimates according to Table 1.

As can be seen, some of the friction parameters have a quite large standard deviation, which actually indicates that the model cannot fully describe the data sets used for identification. The parameters in the right column only affect the low velocity region, and in this region the five data sets with different amplitudes behave quite differently (for example smaller $\beta$ for larger amplitudes). An even more advanced friction model which handles these differences is therefore needed in order to fully capture the friction dynamics.

The separable least squares problem has no local minima in the area of interest, as can be seen in Figure 2. The loss function (2) is also relatively insensitive to different values of the two parameters since such differences can be somewhat compensated by the $F_{c s}$ parameter.

To see the differences, two simpler friction models are used as well, where first $F_{c s}$ (and $\alpha$ ) are set to zero (3 parameter model), and secondly using (8) (2 


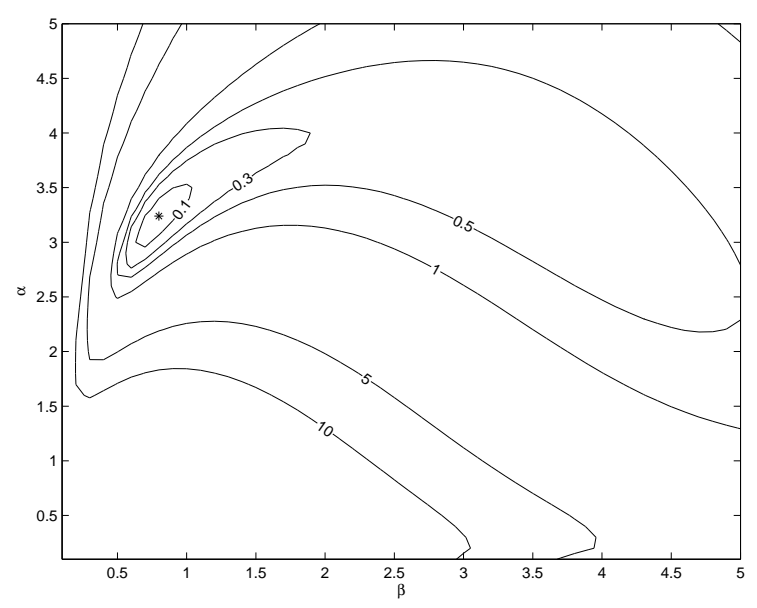

Fig. 2. Level curves showing the increase in the loss function (in percent) when moving away from the optimum.

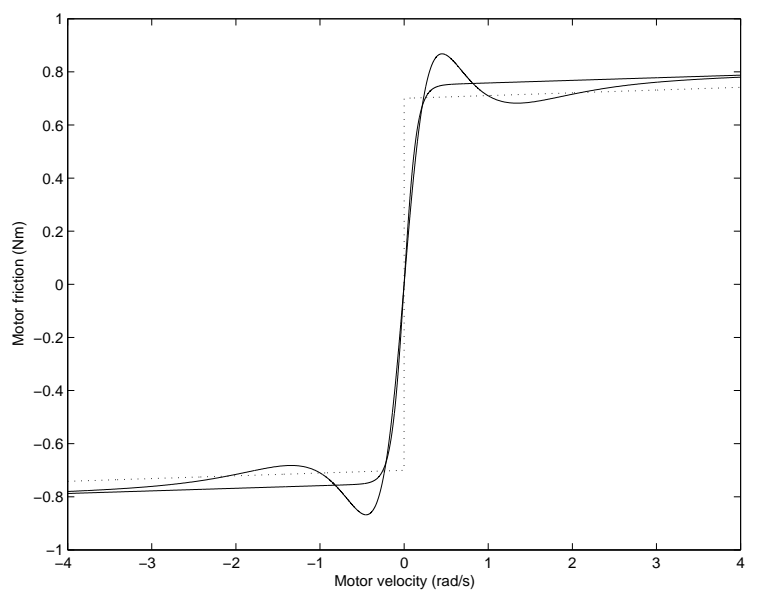

Fig. 3. Estimated friction models. Thick line: (7), thin line: (7) with $F_{c s}=0$, dotted line: (8).

parameter model). Figure 3 shows the resulting friction curves. To evaluate the models, the loss function for some of the amplitudes are shown in Table 2. The advanced model is slightly better than the 3 parameter model. For the 2 parameter model, the loss function for amplitude 1 is drastically increased. For large amplitudes, no major differences can be noted.

\subsection{Step 2}

In this step, first the FRF must be estimated. Before the estimation, the input signal should be modified according to the estimated friction model from step 1. This gives minor differences for data set 2 , but for data set 3 the resonances get more visible. The FRF for data set 2 from motor torque to motor acceleration can be seen in Figure 4 on the next page.

Solving the inverse eigenvalue problem according to Section 4.2 gives initial estimates of $\theta_{r b}=$ $\left(\begin{array}{lll}J_{1} & J_{2} & J_{3}\end{array}\right)^{T}$ and $\theta_{f l}=\left(\begin{array}{llll}k_{g 1} k_{g 3} & k_{a} & d_{g} & d_{a}\end{array}\right)^{T}$, except for $k_{g 3}$ which is kept zero at this step due to the
Table 3. Estimated parameters, where m5init comes from the first two steps and the other models are estimated in step 3.

\begin{tabular}{lllllll}
\hline & m5init & $\mathrm{m} 5$ & $\mathrm{~m} 3$ & $\mathrm{~m} 2$ & $\mathrm{~m} 5 \mathrm{gk} 3$ & \\
\hline$F_{v}$ & 0.986 & 0.986 & 0.979 & 1.04 & 0.986 & $\left(\cdot 10^{-2}\right)$ \\
$F_{c}$ & 0.743 & 0.743 & 0.748 & 0.700 & 0.743 & \\
$F_{c s}$ & 3.99 & 3.99 & 0 & 0 & 3.99 & \\
$\alpha$ & 3.24 & 3.24 & 0 & 0 & 3.24 & \\
$\beta$ & 0.799 & 0.799 & 6.67 & $\infty$ & 0.799 & \\
\hline$J_{m}$ & 5.90 & 8.36 & 8.36 & 8.63 & 8.29 & $\left(\cdot 10^{-3}\right)$ \\
$J_{g}$ & 20.1 & 20.5 & 20.5 & 21.6 & 20.6 & $\left(\cdot 10^{-3}\right)$ \\
$J_{a}$ & 6.87 & 4.03 & 4.06 & 2.65 & 4.06 & $\left(\cdot 10^{-3}\right)$ \\
\hline$k_{g 1}$ & 20.6 & 21.3 & 21.3 & 20.5 & 20.3 & \\
$k_{g 3}$ & 0 & 0 & 0 & 0 & 13.1 & \\
$k_{a}$ & 20.2 & 15 & 15.1 & 11.1 & 15.2 & \\
$d_{g}$ & 62.4 & 50.9 & 51.5 & 50.9 & 46.2 & $\left(\cdot 10^{-3}\right)$ \\
$d_{a}$ & 9.88 & 5.15 & 5.06 & 1.69 & 4.54 & $\left(\cdot 10^{-3}\right)$ \\
\hline
\end{tabular}

Table 4. Average loss when evaluating the models using new realizations of data sets 2 and 3.

\begin{tabular}{llllll}
\hline & m5init & $\mathrm{m} 5$ & $\mathrm{~m} 3$ & $\mathrm{~m} 2$ & $\mathrm{~m} 5 \mathrm{gk} 3$ \\
\hline Data set 2 & 0.5574 & 0.2727 & 0.2746 & 0.3133 & 0.2705 \\
Data set 3 & 0.4441 & 0.1658 & 0.1661 & 0.1656 & 0.1638
\end{tabular}

assumption about linearity. These values can be seen in Table 3, model m5init;

\subsection{Step 3}

Combining the estimates from steps 1 and 2 gives the initial model m5init in Table 3. The nonlinear greybox identification according to Section 4.3 is then applied, using data set 2. First, $k_{g 3}=0$ is used, giving the model m5. Releasing $k_{g 3}$ gives the model m5kg3. For comparison, models are also estimated using the simpler friction models from Section 6.1, giving models $\mathrm{m} 3$ and $\mathrm{m} 2$, where the number indicates the number of friction parameters. The rigid body parameters, $\theta_{r b}$, and the second damper are estimated with a standard deviation less than $10 \%$. For the remaining parameters in $\theta_{f l}$, the standard deviation is less than $2 \%$.

To validate the estimated models, four additional realizations of the multisine signal have been applied, both for data set 2 and 3 . The loss function for each of the models in Table 3 , including the rigid model from step 1 have then been calculated for each of the realizations. Their values have first been normalized by the loss using the rigid model, and secondly averaged over the realizations to get the average loss, shown in Table 4. Comparing m5init and $\mathrm{m} 5$ shows the big improvement by using step 3 in the procedure. For data set 2, the more advanced friction model (7) reduce the loss function, compared to using (8). For data set 3 , on the other hand, there are no major differences. This can partly be understood by the fact that a correct friction model is more important for open loop simulations if one must track a slowly varying signal, like the square wave. Using acceleration as output from the model would probably give a different 


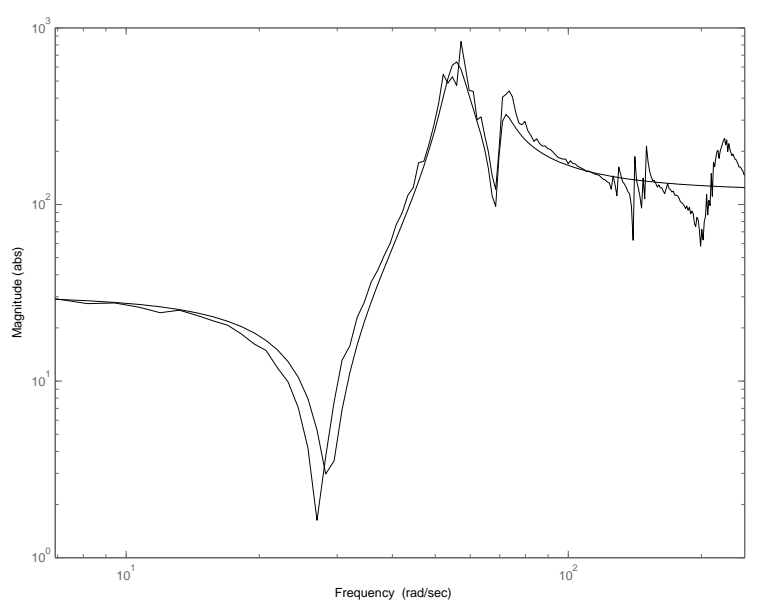

Fig. 4. Magnitude of the FRF for data set 2 from motor torque to motor acceleration (thin line) together with the estimated model m5 (thick line).

result. The nonlinear spring stiffness reduces the loss function, as can be seen by comparing the loss for $\mathrm{m} 5$ and $\mathrm{m} 5 \mathrm{~kg} 3$. Using a data set with a different amplitude would probably show an even bigger difference.

The discontinuous friction model (8) gives problems in simulation and therefore a large $\beta$ value is used instead. This is actually another reason for using the more advanced friction model (7), besides that it gives better performance.

Finally, a Bode diagram of the estimated model m5 can be seen in Figure 4 together with $\mathrm{m} 5$ init and the estimated FRF for data set 2.

\section{CONCLUSIONS}

A three-step identification procedure has been used for the identification of rigid body dynamics, friction, and flexibilities, only using measurements on the motor side. The procedure has been exemplified using experimental data from an industrial robot together with a flexible three-mass model where nonlinear spring stiffness and a nonlinear friction model have been added. The estimated physical parameters have realistic numerical values and give a model with good correspondence to FRF measurements.

To further improve the results the friction could be modeled even more accurately. The method will also be applied to a multivariable system.

\section{REFERENCES}

Albu-Schäffer, A. and G. Hirzinger (2001). Parameter identification and passivity based joint control for a 7DOF torque controlled light weight robot. In: Proceedings of the 2001 IEEE International Conference on Robotics and Automation. Seoul, Korea. pp. 2852-2858.
Armstrong-Hélouvry, $\quad$ B., $\quad$ P. $\quad$ Dupont and C. Canudas de Wit (1994). A survey of models, analysis tools and compensation methods for the control of machines with friction. Automatica 30(7), 1083-1138.

Behi, F. and D. Tesar (1991). Parametric identification for industrial manipulators using experimental modal analysis. IEEE Transactions on Robotics and Automation 7(5), 642-652.

Berglund, E. and G. E. Hovland (2000). Automatic elasticity tuning of industrial robot manipulators. In: Proceedings of the 39th IEEE Conference on Decision and Control. Sydney, Australia. pp. 5091-5096.

Gautier, M. and P. Poignet (2001). Extended kalman filtering and weighted least squares dynamic identification of robot. Control Engineering Practice 9(12), 1361-1372.

Golub, G. and V. Pereyra (1973). The differentiation of pseudo-inverses and nonlinear least squares problems whose variables separate. SIAM Journal on Numerical Analysis 10(2), 413-432.

Grotjahn, M., M. Daemi and B. Heimann (2001). Friction and rigid body identification of robot dynamics. International Journal of Solids and Structures 38, 1889-1902.

Isaksson, A., R. Lindkvist, X. Zhang, M. Nordin and M. Tallfors (2003). Identification of mechanical parameters in drive train systems. In: IFAC System Identification Symposium SYSID 2003.

Johansson, R., A. Robertsson, K. Nilsson and M. Verhaegen (2000). State-space system identification of robot manipulator dynamics. Mechatronics 10(3), 403-418.

Kozlowski, K. (1998). Modelling and identification in robotics. Advances in Industrial Control. Springer. London.

Ljung, L. (1999). System Identification: Theory for the User. 2nd ed. Prentice Hall. Upper Saddle River, New Jersey, USA.

Ljung, L. (2003). System Identification Toolbox User's Guide. The MathWorks Inc. Sherborn, MA, USA.

Pintelon, R. and J. Schoukens (2001). System identification: a frequency domain approach. IEEE Press. New York.

Swevers, J., C. Ganseman, D. B. Tükel, J. De Schutter and H. Van Brussel (1997). Optimal robot excitation and identification. IEEE Transactions on Robotics and Automation 13(5), 730-740.

Wernholt, E. and S. Gunnarsson (2005). Nonlinear grey-box identification of industrial robots containing flexibilities. In: Proc. 16th IFAC World Congress. Prague.

Östring, M., S. Gunnarsson and M. Norrlöf (2003). Closed-loop identification of an industrial robot containing flexibilities. Control Engineering Practice 11, 291-300. 


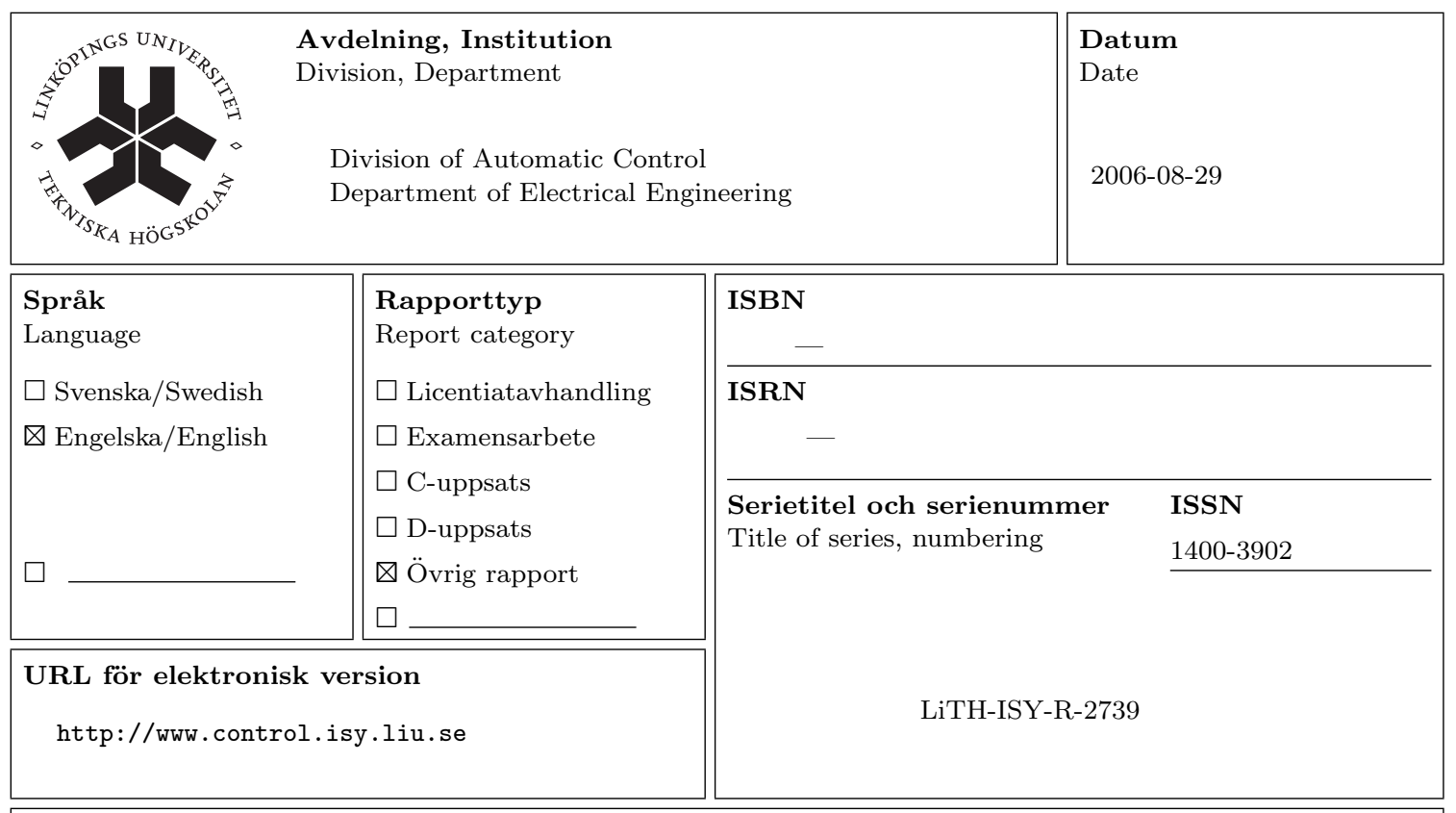

Titel Nonlinear Identification of a Physically Parameterized Robot Model

Title

Nonlinear Identification of a Physically Parameterized Robot Model

Författare Erik Wernholt, Svante Gunnarsson

Author

\section{Sammanfattning}

Abstract

In the work presented here, a three-step identification procedure for rigid body dynamics, friction, and flexibilities, introduced in (Wernholt and Gunnarsson, 2005), will be utilized and extended. Using the procedure, the parameters can be identified only using motor measurements. In the first step, rigid body dynamics and friction will be identified using a separable least squares method, where a friction model describing the Striebeck effect is used. In the second step, initial values for flexibilities are obtained using inverse eigenvalue theory. Finally, in the last step, the remaining parameters of a nonlinear physically parameterized model are identified directly in the time domain. The procedure is exemplified using real data from an experimental industrial robot. 